\title{
Observing desert dust devils with a pressure logger
}

\author{
R. D. Lorenz \\ Johns Hopkins University Applied Physics Laboratory, Laurel, MD 20723, USA \\ Correspondence to: R. D. Lorenz (ralph.lorenz@jhuapl.edu)
}

Received: 2 July 2012 - Published in Geosci. Instrum. Method. Data Syst. Discuss.: 17 July 2012

Revised: 12 November 2012 - Accepted: 13 November 2012 - Published: 4 December 2012

\begin{abstract}
A commercial pressure logger has been adapted for long-term field use. Its flash memory affords the large data volume to allow months of pressure measurements to be acquired at the rapid cadence $(>1 \mathrm{~Hz})$ required to detect dust devils, small dust-laden convective vortices observed in arid regions. The power consumption of the unit is studied and battery and solar/battery options evaluated for long-term observations. A two-month long field test is described, and several example dust devil encounters are examined. In addition, a periodic $(\sim 20 \mathrm{~min})$ convective signature is observed, and some lessons in operations and correction of data for temperature drift are reported. The unit shows promise for obtaining good statistics on dust devil pressure drops, to permit comparison with Mars lander measurements, and for array measurements.
\end{abstract}

\section{Introduction}

Dust devils (e.g., Balme and Greeley, 2006) are vertical convective vortices encountered in arid regions, in particular during periods of strong solar heating (early afternoon in summer). The vortical structure is rendered visible by lofted dust, which may (via sunlight absorption) itself contribute to the strength of the convection intensity.

Pressure drops in dust devils have been noted in the past on Earth (e.g., Wyett, 1954; Lambeth, 1966; Sinclair, 1973), but are actually more systematically documented in studies of dust devils on Mars (e.g., by Mars Pathfinder: Murphy and Nelli, 2002, and by the Phoenix lander, Ellehoj et al., 2010), where landers have recorded meteorological parameters over long periods with a high enough cadence to detect small vortical structures. A number of dust-devil type vortices were detected by the Viking lander (via wind speed and direction, e.g., Ryan and Lucich, 1983 - the pressure measurements were acquired too infrequently to be of use in this application). Interest in further measurements remains high - various proposals for network missions (including pressure sensing) have been made such as NASA's MESUR, the French Netlander, and recently the Finnish METNET. The NASA rover Curiosity, which landed on Mars in August 2012, carries a meteorology package, as will the recently-selected NASA Insight mission to be launched in 2016. All these missions may detect dust devils on Mars via pressure records and other data.

Routine terrestrial meteorological stations only record data at $\sim 15$ min cadence, too infrequently to detect dust devils. As discussed in Lorenz (2012), it would be highly desirable to obtain a dataset of $\sim 1 \mathrm{~Hz}$ or better fixed-station pressure data at a place and time where dust devils are known to occur. Such a dataset should last several weeks (Mars experience suggests of order one encounter per day can be expected, so several months of operation of a single station are needed) in order to obtain a useful number of dust devil encounters (i.e., of order a hundred or more, to permit robust comparison with Mars). While mobile measurement platforms (e.g., Sinclair, 1973) can obtain a larger number of encounters in a given time, they do so at considerable expense in labor, and at the cost of introducing selection bias in the dust devils encountered (largest, slowest, etc.) and with possible vehicle effects on part or all of the record.

Sites with high dust devil activity tend to be hot and remote, and long-term unattended operation presents hazards of theft or vandalism. Certain controlled areas where equipment could be supervised (e.g., military bases) may be feasible but present access challenges. Another approach, although not easy with conventional meteorological masts, is to deploy compact instrumentation with a minimal visual signature, such that it is unlikely to be detected and thus interfered with. Recent technological developments in precision 
pressure sensors and flash memory allow such compact and thus discreet sensing platforms to be deployed for extended periods with a good probability of recovery, and at a cost wherein a finite probability of loss is tolerable.

\section{Pressure logger}

\subsection{Commercial unit as supplied}

The commercial pressure logger used here is the B1100-1 logger by Gulf Coast Data Concepts. The unit (Fig. 1) is essentially a small circuit board with a USB connector for data transfer: all components (including the pressure sensor, a reset switch, and a holder for a AA battery) are mounted on this board, which is protected by a three-part semitransparent plastic housing. The housing is about $2.5 \mathrm{~cm}$ in diameter and $10 \mathrm{~cm}$ long: with the AA battery installed, the unit weighs only $55 \mathrm{~g}$.

The product literature (GCDC, 2010) reports that the unit will run for 3 weeks on an alkaline AA cell. This, however, appears to be only at a low sample rate. A requirement for detecting small dust devils, distinct from more general meteorological investigations, is that samples be acquired at a high rate of one sample per second or better. Our initial tests showed that an alkaline AA cell will last only a few days at the highest rate of $10 \mathrm{~Hz}$, although at $1-2 \mathrm{~Hz}$ sampling about 10 days of continuous operation can be expected.

The logger stores readings acquired at rate up to $10 \mathrm{~Hz}$ in a comma-delimited text file (CSV), with a timestamp for each reading. These files are written as ASCII text files to a 2 GB micro-SD flash memory card. This card is also used to hold a configuration file which determines the sample rate, file size, etc. The sensor (Bosch Sensortech, 2009) in the unit is a BMP-085 Digital Pressure Sensor by Bosch Sensortec, in a $5 \times 5 \times 1.2 \mathrm{~mm}$ package which communicates with the B1100 microcontroller using an I2C interface and has a nominal resolution of $0.01 \mathrm{mb}$. The BMP-085 is factory-programmed with 11 16-bit constants that are used, together with an on-chip temperature measurement, by the microcontroller to correct the raw pressure reading into a calibrated value.

\subsection{Data storage}

The ASCII file written by the loggers is less compact than a binary format, but considerably more convenient in most applications. A header contains the unit serial number, the file origination time, battery voltage and setting information, then a user-specified number of measurement lines follow, with each line containing the measurement time in decimal seconds after the file origination time, and the pressure reading in Pascals as an integer, and optionally (see below) an integer temperature reading in tenths of a degree Celsius.

A file with 86400 measurements (i.e., a day of data at 1 sample per second) occupies $1.5 \mathrm{MB}$ : even at the maximum

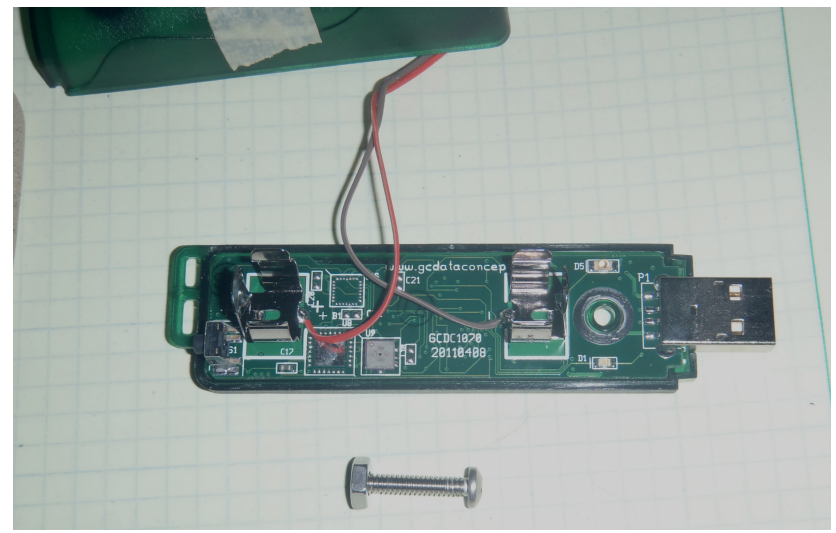

Fig. 1. Datalogger with the casing partly removed. The USB connector is at right, the AA battery terminals are visible at right and left (with user-supplied wires soldered there to provide an alternative power supply in this application). The pressure transducer is the silver square at the lower edge of the board. The memory, microcontroller and other components are on the other side of the circuit board.

sample rate of $10 \mathrm{~Hz}$, about 4 months of data could be stored in the $2 \mathrm{~GB}$ card supplied with the unit. Note that the filesystem on the logger only records 999 data files. While large datafiles can be inconvenient (e.g., at least some versions of Microsoft Excel have difficulties plotting more than 32768 points, and code in IDL or a similar language needs loop or index variables to be of long integer type), it may be necessary to use large files if high sample rates are intended for long periods of observation in order to keep the total number of files below 1000 .

Several additional pieces of information are stored. First, the battery voltage is recorded in the header of the file. Secondly, every 100 pressure readings (or at some other interval - it is specified by the user in the configuration file) a temperature reading is written. Since the temperature sensor is simply on the circuit board, it has too high a thermal inertia to respond quickly to air temperature changes and thus is not of particular interest in dust devil measurements directly. Including the temperature data adds five characters to each line (a three digit temperature reading plus a space and comma), which typically is $12-16$ characters long with the time and pressure reading, i.e., it imposes a $20-30 \%$ data overhead; thus, to include the temperature data at every reading would reduce the number of pressure readings that could be accommodated in the flash memory. Thus, since the temperature data were not expected to be of immediate meteorological interest, an interval of 100 readings was chosen; we will return to this decision later.

The file header contains a time-tag from a real-time clock (set by the user). The time increment after this tag is recorded for each pressure measurement as a decimal value in seconds, so the absolute time of each reading can be calculated readily. Thus, data from different pressure loggers can 


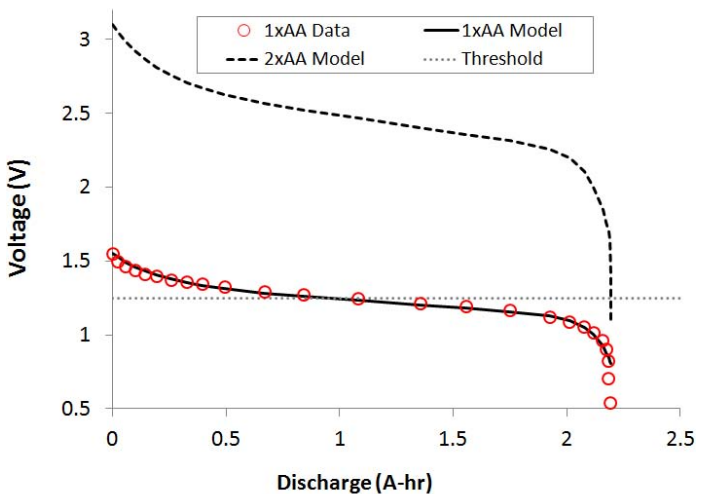

Fig. 2. Discharge curve of a Duracell alkaline AA cell $(50 \mathrm{~mA}$ constant current) from manufacturer's datasheet (circles), compared with a simple analytic model $C=\mathrm{Co} /[1+\exp \{(V-V o) / \mathrm{d} V\}]$ with $\mathrm{Co}=2.2 \mathrm{~A}-\mathrm{h}, \mathrm{Vo}=1.25 \mathrm{~V}$ and $\mathrm{d} V=0.07 \mathrm{~V}$ (solid line). Where this curve intersects the voltage threshold of the logger (dotted line), the unit stops - in this case (threshold $=1.25 \mathrm{~V}$ ) after a discharge of about $1 \mathrm{~A}-\mathrm{h}$ - even though substantial capacity remains. One mitigation is to put two cells in series (dashed curve) - here the curve intersects the threshold after some $2.2 \mathrm{~A}-\mathrm{h}$. The rather shallow discharge curve of alkaline cells means that if the threshold is even slightly higher, a significant drop in useful battery capacity results.

be synchronized with modest effort and care in setting the clock for each unit. Since each clock can be set from the same PC, an initial synchronization to about $1 \mathrm{~s}$ should be achievable (no attempt has been made to evaluate the clock performance.)

\subsection{Power consumption}

It was noted in initial tests that the logger ceased operation when the battery voltage fell to about $\sim 1.25 \mathrm{~V}$. For conventional alkaline batteries, this in fact means there is considerable capacity left in the cell (see Fig. 2). Inquiries with the manufacturer indicated that a $\sim 1.2 \mathrm{~V}$ cut-off was introduced to avoid brownout of the system during file-write operations to the SD card, when the current draw can increase momentarily up to $100 \mathrm{~mA}$. Corruption of the file system can result if the voltage drops too low under this current draw, and thus operations are suppressed when the voltage is low enough that this might be a risk. In fact, we have noted similar problems (and in fact introduced a similar precaution to cease operations before battery voltage fell too low) in experiments with digital time-lapse cameras used to study dust devils (Lorenz et al., 2010).

The device (Alex Kooney, personal communication, 2012) uses a boost regulator to generate a $3.3 \mathrm{~V}$ operating voltage from the battery supply. Because of this boost operation, current consumption decreases for higher battery voltage, which can be higher than $1.5 \mathrm{~V}$ (although no advantage is gained above $3.3 \mathrm{~V}$ ). We have measured the current consumption for $1.5 \mathrm{~V}$ and $3.0 \mathrm{~V}$ supplies and list the results in Table 1;
Table 1. B1100-1 Current consumption for one fresh alkaline cell and two cells in series.

\begin{tabular}{lrrc}
\hline $\begin{array}{l}\text { Samples } \\
\text { per } \\
\text { second }\end{array}$ & $\begin{array}{r}\text { Current } \\
\text { draw } \\
(\mathrm{mA})\end{array}$ & $\begin{array}{r}\text { Current } \\
\text { draw } \\
(\mathrm{mA})\end{array}$ & $\begin{array}{c}\text { Current } \\
\text { draw } \\
(\mathrm{mA})\end{array}$ \\
$\begin{array}{r}1.5 \mathrm{~V} \\
\text { supply } \\
\text { LEDs on }\end{array}$ & $\begin{array}{r}1.5 \mathrm{~V} \\
\text { supply }\end{array}$ & $\begin{array}{c}3.0 \mathrm{~V} \\
\text { LEDs off } \\
\text { LEDply on }\end{array}$ \\
\hline 1 & 7.0 & 6.7 & 4.0 \\
2 & 7.4 & 7.3 & 4.0 \\
5 & 11.0 & & 7.7 \\
3.3 & 15.4 & & \\
10 & 17.0 & 16.0 & 9.3 \\
\hline
\end{tabular}

indeed, for a given sample rate the current draw is reduced by a factor of just under two for the higher supply voltage. The unit's configuration file also allows the user to disable the indicator LEDs - this change makes only a modest $(\sim 5 \%)$ difference in consumption. For the $1.5 \mathrm{~V}$ supply, the current varies roughly linearly $I=6+1.1 \mathrm{~s}$, where s is the sample rate in $\mathrm{Hz}$ and $I$ the current in $\mathrm{mA}$. Note that the currents in Table 1 are the typical values; there are brief spikes during flash write operations that are not accounted for.

Thus, our approach to enhancing the longevity of the system is threefold. First, two alkaline cells are used in series. This reduces the current consumption due to the (typically) higher supply voltage, which introduces a factor of nearly two increase in endurance. Second, because the discharge curve of two cells in series only reaches $1.25 \mathrm{~V}$ when the cells have been almost completely depleted $(\sim 2.1 \mathrm{~A}-\mathrm{h}$, compared with $\sim 0.8$ A-h for a single cell to $1.25 \mathrm{~V}$, see Fig. 2), an additional factor of $\sim 2.5$ is obtained. Finally, larger $(D)$ cells are used, bringing a final additional factor of $\sim 6-10$ over AA cells. Thus, the total endurance is enhanced by $\sim 30$ compared with the single AA cell by the substitution of 2-D cells with a battery holder, totaling about $\$ 5$ per unit. The logger and $D$ cells/holders fit inside a "2.5-cup" polypropylene food container, sprayed with sand-textured paint for this application (see Fig. 3). Note that a hole must be drilled in this waterproof housing to permit external pressure changes to be rapidly communicated to the sensor and to prevent temperature changes causing the internal pressure to vary.

An obvious alternative approach is to use solar power - especially since the object of the study is areas that are subject to strong solar heating. A plethora of options exist. Simplest is to substitute solar power when available for primary (alkaline) power, but since the device also typically runs at night (actually optional - the configuration file allows for operation between designated times), this only reduces the consumption by about half. Better is to charge a secondary (rechargeable) battery during the day to allow the energy from the solar cells to be exploited for night use as well. For overall energy balance, this requires a solar cell current that exceeds 


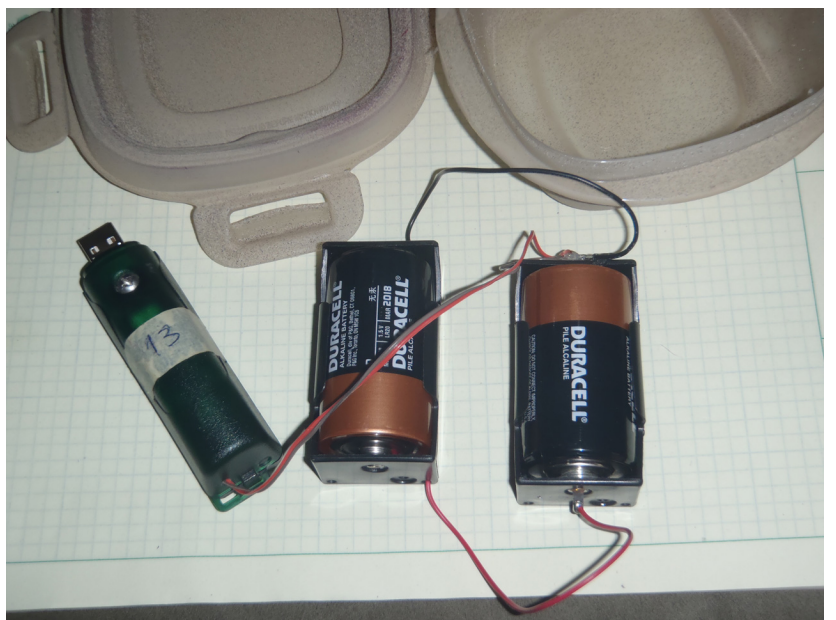

Fig. 3. Sand-colored waterproof food container used to house 2-D cells and the pressure logger for field measurements.

the power consumption by a factor of 3-4. Because extended cloudy periods may allow the secondary battery to become temporarily exhausted (which would reset the datalogger and stop its operation), this approach was adopted in parallel with a pair of alkaline AA cells as backup. This is particularly important since Nickel Cadmium (NiCd) rechargeable cells perform poorly at the very high temperatures encountered by dust devil survey instrumentation. The solar cell charged a set of $3 \mathrm{NiCd}$ AA cells (thus a nominal 3.6 V dropped to $3 \mathrm{~V}$ at the logger power terminals by a $1 \mathrm{~N} 4148$ diode in series; a similar diode in series with the alkaline cells isolated them unless the NiCd voltage fell below $2.4 \mathrm{~V}$ ).

\subsection{Design for measurement campaign}

We now have the information at hand to best configure the system for field use. For a 1 week campaign, a single AA cell will suffice if $2 \mathrm{~Hz}$ data is adequate; for $10 \mathrm{~Hz}$ data $2 \times \mathrm{AA}$ or $1 \times D$ cell must be used. For a 1 month campaign, $2 \times \mathrm{AA}$ or $1 \times D$ will allow $2 \mathrm{~Hz}$ data, but the $2 \times D$ option must be exercised for $10 \mathrm{~Hz}$ data. For a 4-month campaign, the $2 \times D$ cell (or solar) options are needed, and will permit $10 \mathrm{~Hz}$ data. Note, however, in these cases and for longer measurements, the data storage becomes the limiting factor. As indicated in Sect. 2.2 above, 4 months of operation at $10 \mathrm{~Hz}$ will fill the 2 GB memory. Note also that 4 months ( $\sim 1 E 7 s)$ corresponds to 1E8 lines of data, so the 1000-file limit of the file system would require that each file exceed 100000 lines in length.

\section{Field trial}

Three units were deployed on 16 April 2012 on a playa (dry lake bed) in southern Nevada (Fig. 4), a location known (e.g., Pathare et al., 2010) to see dust devil activity. Each was set at the foot of a small sagebrush (Fig. 5), which provided some

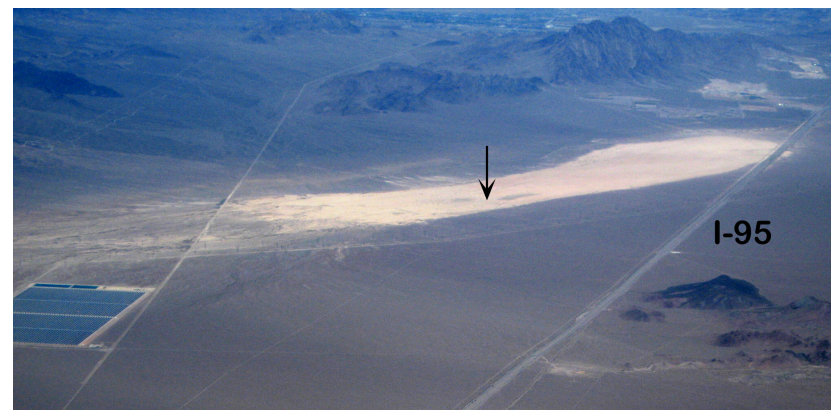

Fig. 4. The playa (about $6 \mathrm{~km}$ long) seen from a commercial airliner approaching Las Vegas. The approximate measurement site is arrowed. Highway I-95 runs roughly north-south. The blueish rectangular feature at left is a solar power facility.

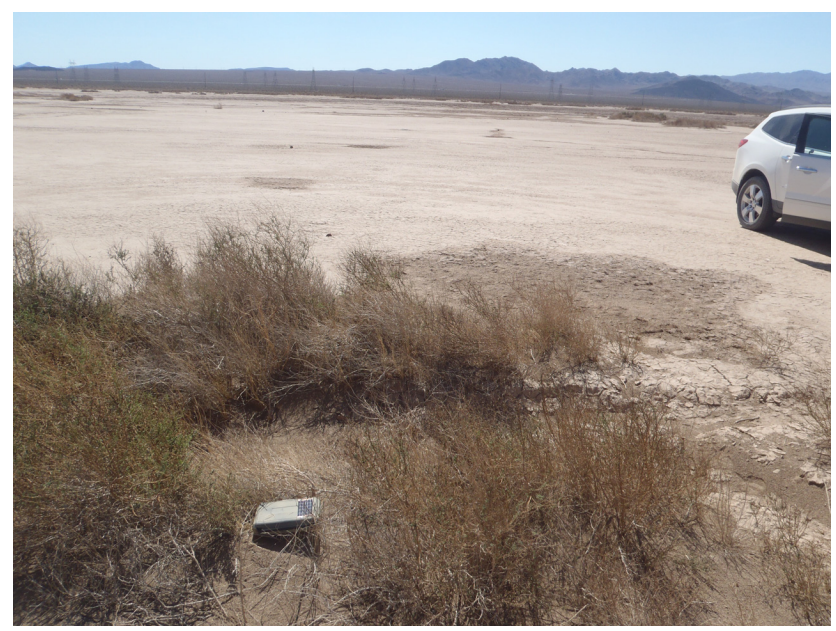

Fig. 5. Sagebrush patch on the playa with solar-powered datalogger installed.

shelter from wind and some concealment, and the location recorded with GPS. Two units ("P10" and "P16") were powered by alkaline D-cells, and the other ("P15") by three AA $\mathrm{NiCd}$ cells recharged with a solar cell and with a 2 AAalkaline cell backup. The units were left unattended until recovered on 21 May 2012; the initial measurement period was therefore 35 days. At this point data were downloaded, and another battery unit ("P12") was deployed and operations continued for another three weeks until the surviving units were recovered on 11 June 2012.

All three units operated successfully for the first measurement duration and some $2 \mathrm{Gbit}$ of data were obtained. Contemporaneous hourly meteorological data were obtained from the Remote Automatic Weather Station (RAWS) at Red Rock Canyon, about $10 \mathrm{~km}$ to the northeast, which is not discussed further in the present paper.

Datafiles were written with lengths of 32000 samples and 86400 samples, with about 970 files and 360 files, respectively. The large number of files allows a good record of the battery voltage history (see Fig. 6). 

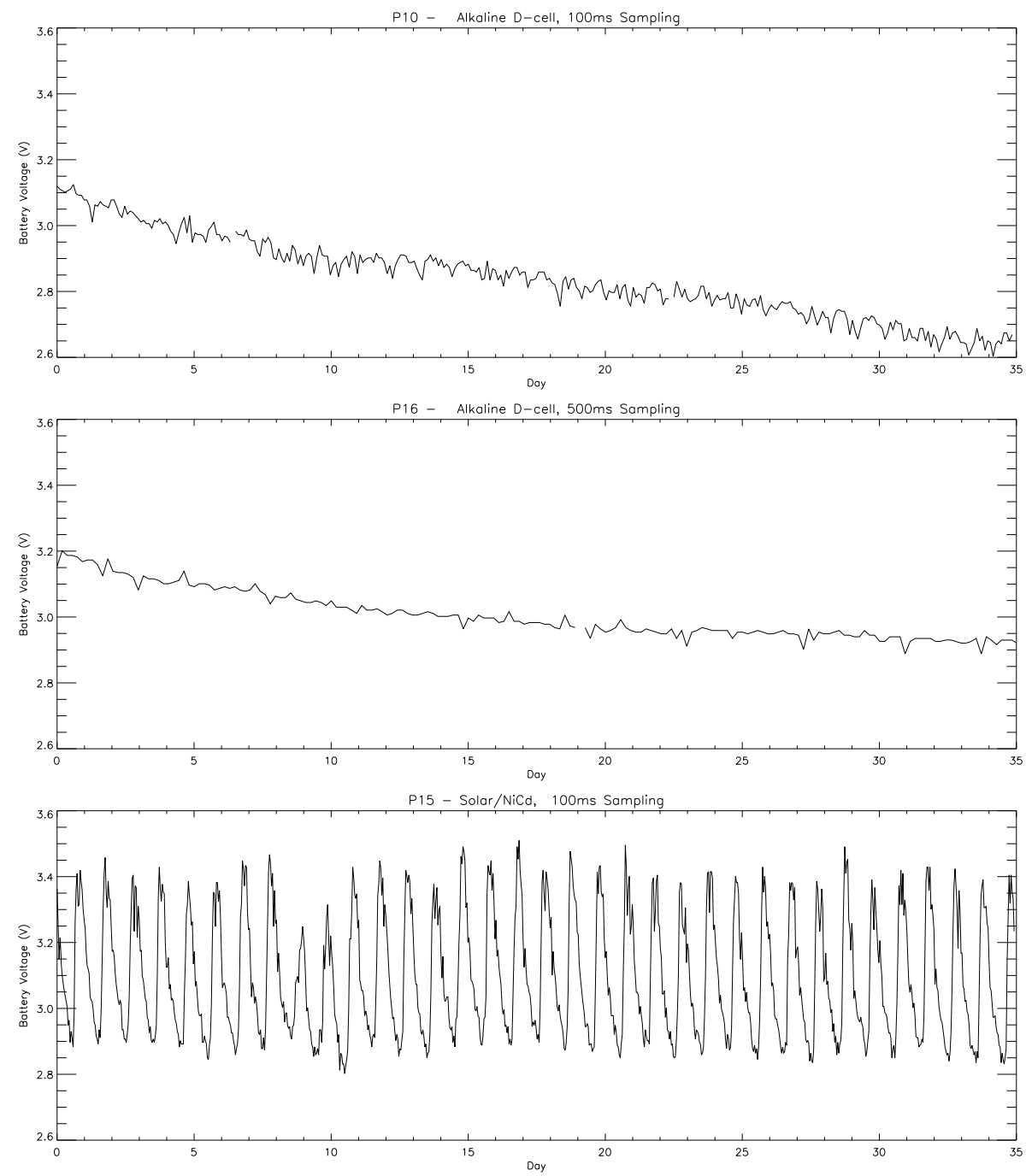

Fig. 6. Battery voltage at the start of each datafile recorded by the three sensors in April 2012. Note the steep initial decline (compare with Fig. 2) for the top two alkaline-only units. Note the slower decline for P16, with the lower sample rate. The lowest unit shows a generally uniform diurnal cycle, although peak voltage dropped on days 9 and 10 due to clouds.

Three units and another $\sim 2 \mathrm{~GB}$ of data were obtained when the site was visited in early June 2012. Unfortunately the solar-powered unit was not present, presumably removed by unknown persons (the playa is frequented for various recreational activities, including drag racing, flying radio controlled airplanes, etc.). Perhaps the necessity of maintaining a clear sky view to obtain solar power made this installation particularly visible to passersby. It may be that the penalty of finite battery lifetime is worth the reduced probability of theft or damage by better concealment (or even burial) that is impossible for solar-powered installations. Similarly, adding a prominent notice "Scientific Equipment, Please Leave Undisturbed" may decrease the probability of interference or removal if the unit is found, but increases the probability of it being noticed in the first place.

\section{Field data and noise performance}

The absolute calibration of the unit is not important for the present application although the manufacturer's data sheets (Bosch Sensortech, 2009; GCDC, 2010) quotes $1 \mathrm{hPa}$ (i.e., $\sim 1 \mathrm{mb}$ ). The noise level depends on the measurement mode and is quoted as $3-6 \mathrm{~Pa}$ or $0.03 \mathrm{mb}$. The reading is output as an integer in $\mathrm{Pa}$ (i.e., a resolution of $1 \mathrm{~Pa}$ or $0.01 \mathrm{mb}$ ). Indoor tests on short sequences of data (where no trend in pressure is obvious) show Gaussian-distributed scatter with a standard deviation of $\sim 3 \mathrm{~Pa}$, suggesting that the specification is met in quiescent conditions.

In addition to features in the pressure data interpreted to be dust devils (see Sect. 5), some other aspects of the field data were noted (see Fig. 7). While in general the noise characteristics of the data were comparable with the indoor 

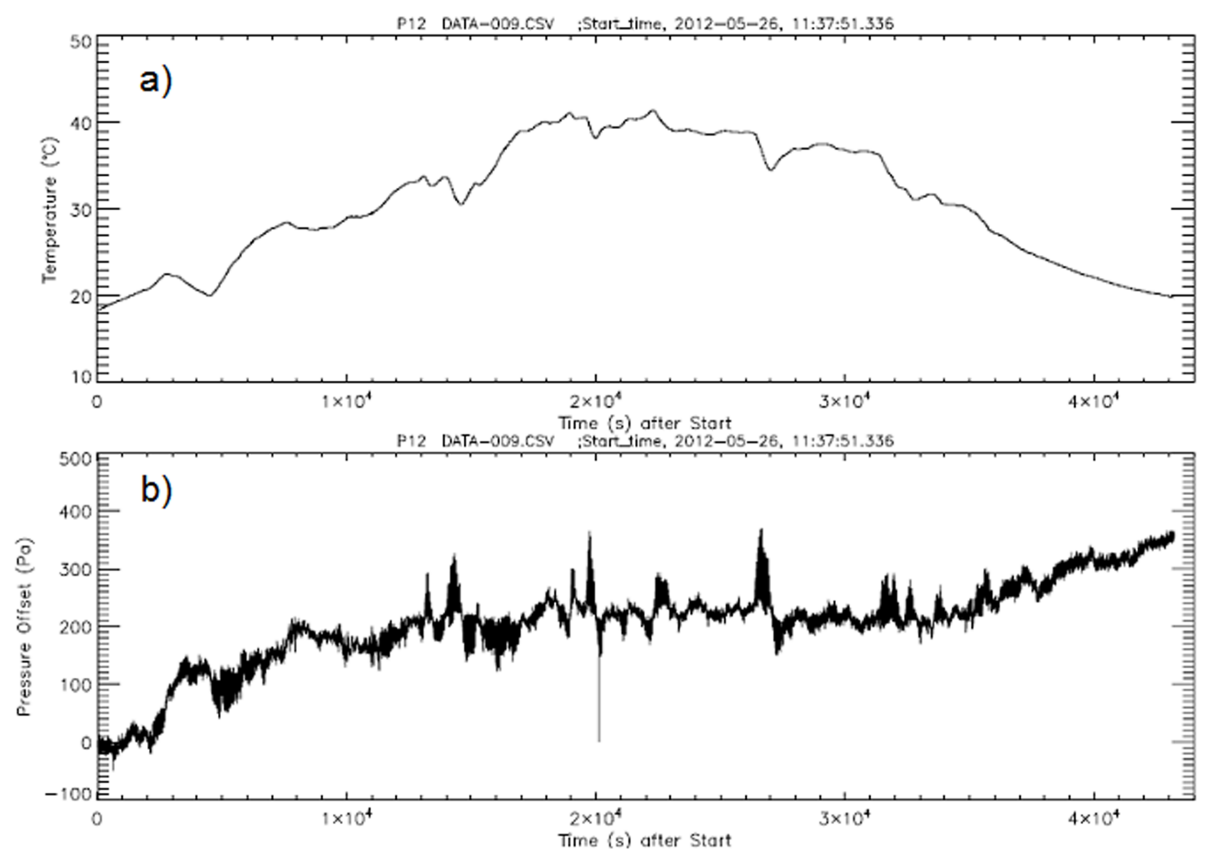

Fig. 7. Example data from a logger ("P12") during a follow-up measurement campaign, showing about $12 \mathrm{~h}$ of data. (a) Sensor temperature rises throughout the day to a peak (in the shade) of $40^{\circ} \mathrm{C}$. Note that dips in temperature occur at $\sim 5000 \mathrm{~s}$ intervals. The timestamp on the data files is Eastern Daylight Time (from the author's laptop), which is $3 \mathrm{~h}$ ahead of local time (Pacific). Thus, the record shown here begins at 08:38 a.m. LT (local time). (b) Pressure record, with an initial offset subtracted. Fluctuations of $\sim 10$ Pa are seen throughout, but some isolated bursts of $\sim 100 \mathrm{~Pa}$ noise are seen, correlating with the temperature dips; the instance at $27000 \mathrm{~s}$ is shown in more detail in Fig. 8 .

trials indicated above, some dramatically poorer behaviors were also noted. These have been studied here in order to improve them for future measurements, and in particular to improve the performance of automatic dust devil detection algorithms.

First, a sawtooth pattern (Fig. 8) was sometimes apparent in the data - corresponding to what looks like noise in the daily view (Fig. 7). This did not degrade the data quality dramatically beyond the normal noise level for the two loggers that ran with $100 \mathrm{~ms}$ sample interval, but the third unit with a sample interval of $500 \mathrm{~ms}$ showed a much more pronounced sawtooth. Interestingly, the scatter in the data for that unit within each sawtooth cycle was noticeably smaller than for the other two, suggesting that perhaps the highest sample rate causes higher measurement noise. The sawtooth cycle was a very regular 100 samples, which was the arbitrary value chosen as a compromise between the limited expectation of utility of the temperature data and the data volume cost of recording it. Thus, it was suspected upon examination of the data that the temperature recording is actually used in the temperature correction of the pressure sensor data, and that the sawtooth (most noticeable during the day) was due to comparatively rapid temperature changes such that the correction was not being correctly applied. An obvious mitigation strategy is to simply decrease the interval between temperature readings to just a few seconds (i.e., every 10th sample for a $500 \mathrm{~ms}$ pressure sampling cadence, or every 50th for the fastest $100 \mathrm{~ms})$. The improvement in data quality is likely worth the data volume penalty (see Sect. 2.2). Because this temperature calibration error is somewhat deterministic, a correction algorithm can be applied to improve the data quality (Fig. 8). Different filtering options (even simple smoothing) can likely improve the detectability of dust devil pressure drops, especially when the width of the drop being sought is much $(\sim 10 \times)$ wider than the interval between temperature corrections.

Another mitigation (Alex Kooney, personal communication) is to add a "raw-output" flag to the configuration file; this option (not documented in the product manual, GCDC, 2010) forces the device to output a reading that is not "corrected" with an erroneous temperature. While this entails post-processing to recover absolute pressures, or indeed to correct for long-term relative variations, this raw output will vary smoothly without any sawtooth noise, which may be better for detecting short-term dips in pressure due to dust devils.

A related noise effect appears in some instances. This has the appearance of an irregular square wave superposed on the data. This appears to be similar to the sawtooth in origin (in that the steps occur when temperature readings are made) but is due to the finite resolution of the temperature reading (i.e., the error corresponds to a temperature "correction" that uses a value that differs from the true one by less than $0.1{ }^{\circ} \mathrm{C}$; this is the limiting resolution of the BMP-085 onboard sensor, but 

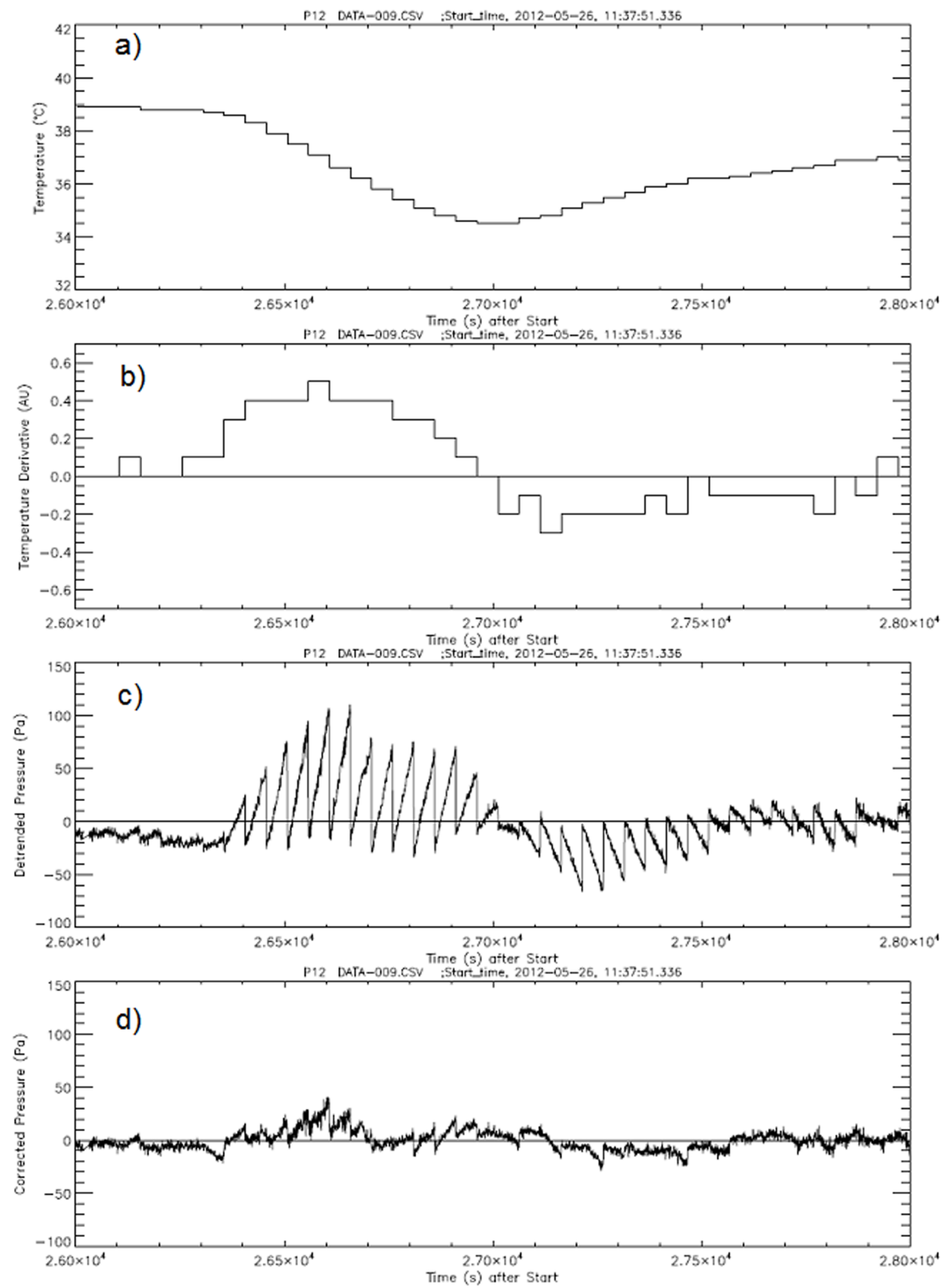

Fig. 8. The $2000 \mathrm{~s}$ of data from Fig. 6 showing (a) the temperature evolution (note $0.1{ }^{\circ} \mathrm{C}$ quantization) and (b) the temperature increment between successive samples (acquired at 100 pressure-sample intervals, or $50 \mathrm{~s}$ ). The pressure readings (c) show a $\sim 100 \mathrm{~Pa}$ sawtooth noise due to improper temperature correction - it is seen that the sawtooth amplitude and sign correlates rather well with the temperature derivative rate. (d) A post-processing correction algorithm subtracts a sawtooth correction (i.e., a cyclic ramp with a period of $50 \mathrm{~s}$, synchronized to the temperature reading) with an amplitude proportional to the temperature derivative in (c): it is seen that the noise is reduced by a significant factor $(\sim 3-4)$ to $\sim 20-30 \mathrm{~Pa}$.

is in fact large enough to give a noticeable pressure error). Significant correction of this noise by post-hoc processing is likely to be challenging, since the information to make the correction simply does not exist (i.e., this noise is somewhat comparable with the residual noise after bad sawtooth has been corrected.) However, as for the sawtooth noise, changing the temperature measurement cadence to a much longer, or (desirably) much shorter, interval will mitigate its impact on dust devil detection.

Finally, an additional data quality issue occurs, in that the text file is occasionally corrupted. Typically a line is truncated, or in most cases a number of spurious " $y$ " characters is introduced. A broadly effective mitigation is to apply robust error-trapping in the code used to read and plot the data and 

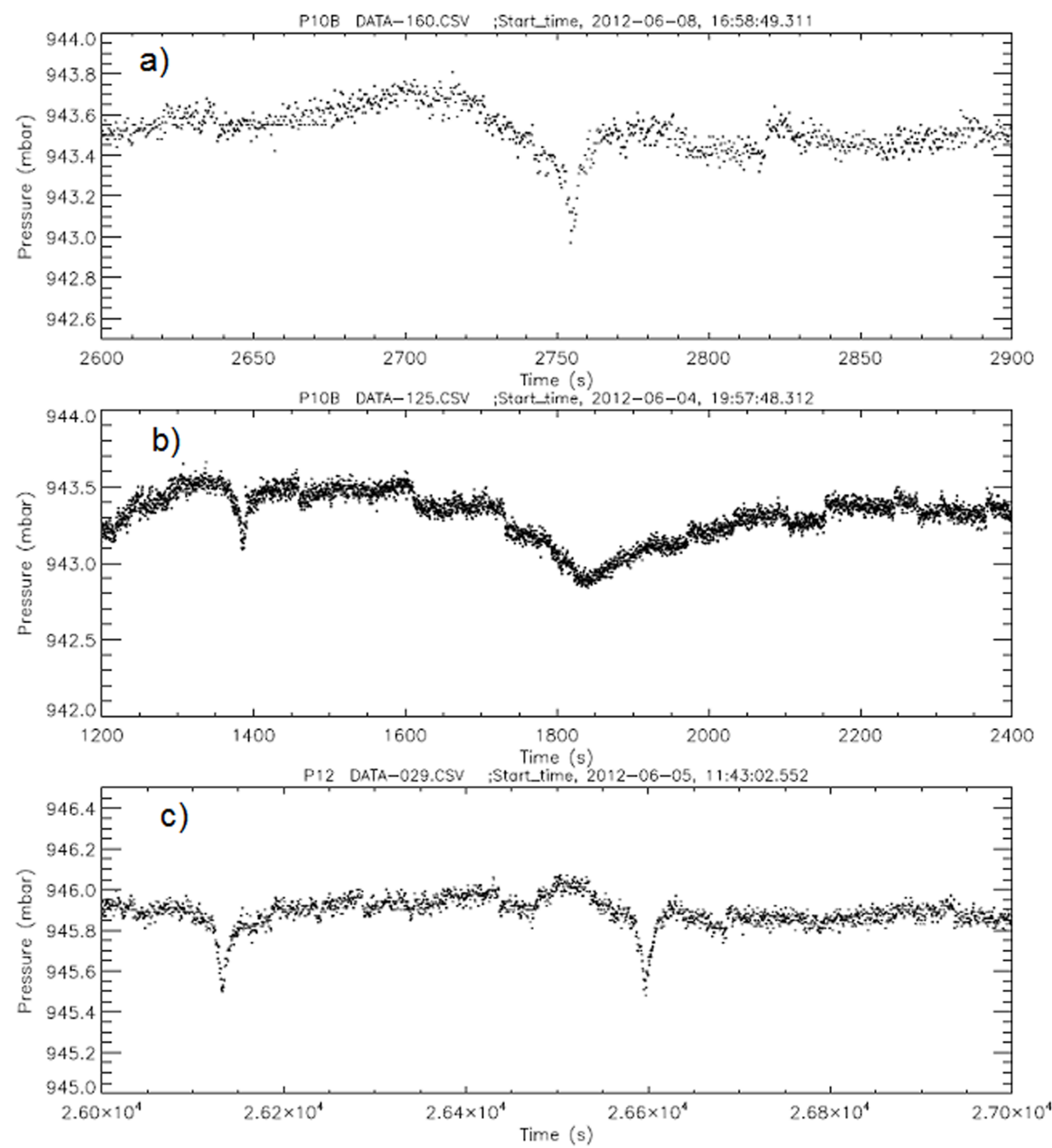

Fig. 9. Some example dust devil signatures. (a) An isolated 30s-wide event with a peak pressure drop of $0.5 \mathrm{mbar}$, (b) a narrow $(\sim 30 \mathrm{~s}$, $0.3 \mathrm{mbar}$ ) and $400 \mathrm{~s}$ later a wide $(300 \mathrm{~s}, 0.5 \mathrm{mbar})$ feature, the latter presumably due to a large devil at some distance. (c) A sequential pair of similarly-sized devils, each $\sim 300 \mathrm{~s}$ wide and $\sim 0.4 \mathrm{mbar}$ deep and separated by $5000 \mathrm{~s}$. Note each plot is scaled differently. Data are uncorrected for sawtooth effects.

reject those lines (the text file can be manually edited, but this would become prohibitively laborious for large amounts of data such as that analyzed here). No obvious time or temperature correlation has been noted with these dropouts (although notably one brief dropout occurs in the middle of a dust devil encounter - see later - suggesting that perhaps nearby electrostatic discharge associated with triboelectric effects in the dust can cause data transfer problems).

While these issues are inconvenient, especially to automated processing of the data, it should be recognized that overall they occur fairly seldom. Many files are unaffected, and in most of those that are, over $99 \%$ of lines in the ASCII record are uncorrupted.

While the noise in the data acquired in this initial field test was somewhat distracting due to the more rigorous temperature excursions experienced than is typical in laboratory or domestic settings, it was nonetheless easy to identify many dust devil signatures by sight. Further, important lessons have been learned for the optimum settings to use for ongoing and future measurements.

\section{Example dust devils and other observations}

A systematic investigation of dust devil population data from the logger described here will be perfomed in future work, using both human and machine methods to detect dust devils. An initial (human) reconnaissance of the data show many "classic" dust devil encounters, and some examples are shown in Figs. 9-11.

Pressure drops in Fig. 9 are 0.3-0.5 mbar deep, with durations of $30-300 \mathrm{~s}$. These examples are fairly symmetric in shape, although usually the dust devil introduces a discontinuity in the background pressure (i.e., the pressure recovers after dust devil passage to a value different from 

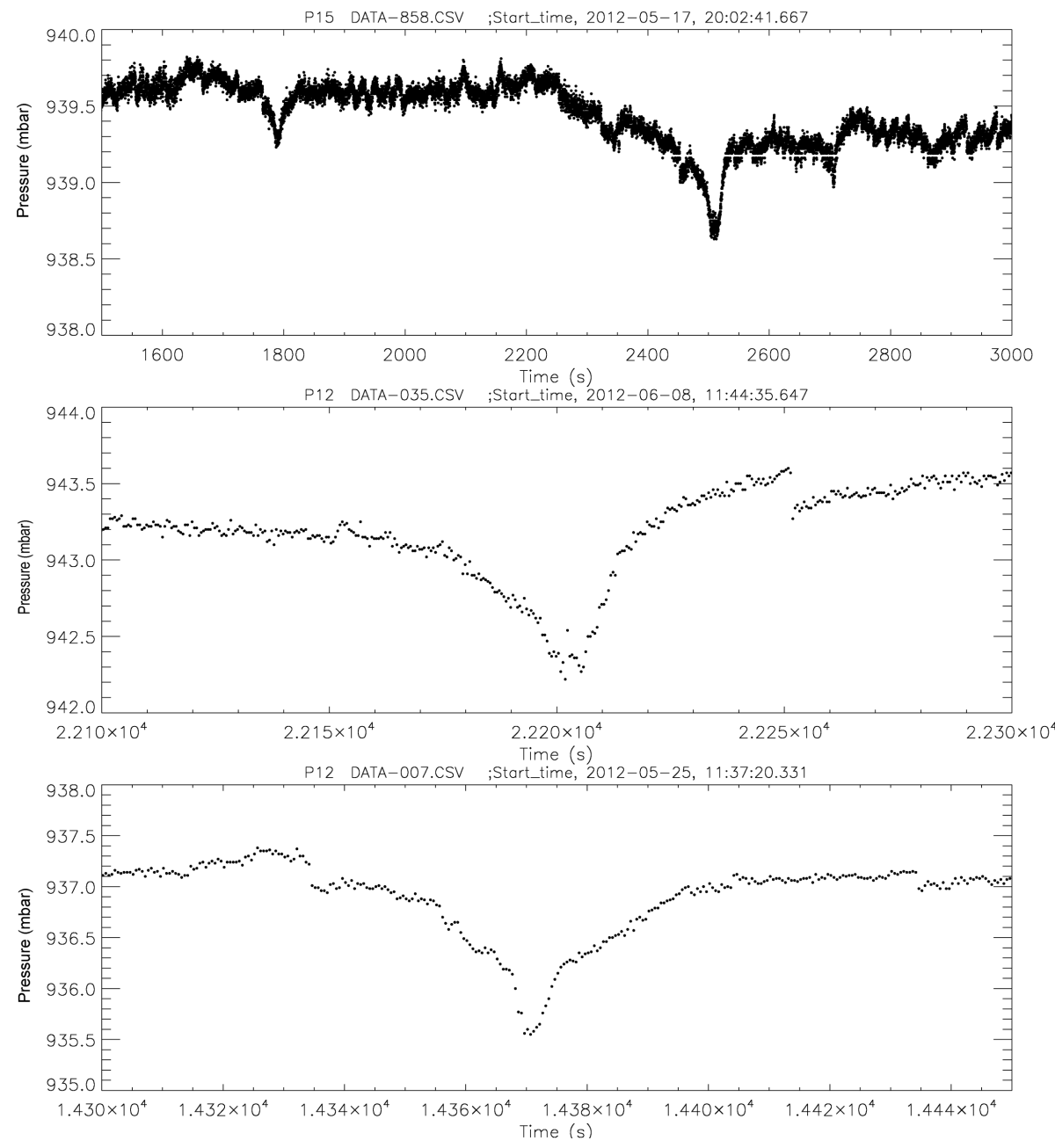

Fig. 10. Further dust devil signatures. (a) A 0.4 mbar $60 \mathrm{~s}$ event and a $0.6 \mathrm{mbar} 100 \mathrm{~s}$ event, spaced by $700 \mathrm{~s}$. (b) A $1 \mathrm{mbar}$ event, $500 \mathrm{~s}$ long, with an apparent double dip in pressure, perhaps due to a core wandering in a cycloidal pattern (c) the largest event noted so far, 1.5 mbar deep, $100 \mathrm{~s}$ wide, with a distinct $0.6 \mathrm{mbar} 10 \mathrm{~s}$-wide core. Note each plot is scaled differently. Data are uncorrected for sawtooth effects.

the pre-encounter value). This was noted, e.g., by Sinclair (1973), although only a handful of examples were shown.

Two of the plots show two dust devils; it is tempting to speculate these may be pairs (e.g., formed by the break of a horizontal roll vortex into two counter-rotating vertical vortices). Future work will explore, with the robust statistics permitted by the large dataset being acquired, the possibility of pairing or other temporal clustering.

Figure 10 shows additional examples. The second event in Fig. 10a is noticeably asymmetric, with a slow dip but a fast recovery. This could be the result of a change in wind speed involving a slow approach with a faster departure for a dust devil that has a constant intensity; or where, for instance, a dust devil suddenly "dies" soon after closest approach. Again, the methodology described in the present paper will permit enough data to be acquired to determine whether such asymmetry (again, noted first by Sinclair, 1973) is random or whether, for example, the pressure decline on the leading face of a devil is shallower than the trailing side. This example also underscores the potential for future measurements with an array of loggers to resolve the temporalspatial ambiguity.

The third example in Fig. 10 is the most intense encounter observed so far, $1.5 \mathrm{mbar}$ deep. Interestingly, it seems to have a distinct broad ramp on either side, a few tens of seconds wide, with a sharp central dip.

Finally, Fig. 11 shows a dual dust devil encounter as observed with three different stations. Stations P15 and P16 were about $50 \mathrm{~m}$ apart, whereas P10 was about $300 \mathrm{~m}$ to the south, which is the direction from which the prevailing wind usually blows. This seems consistent with the closest approach (assumed to be the time of deepest pressure drop) being $\sim 90$ s earlier in $\mathrm{P} 10$ than in P15/16, suggesting a migration speed of $3-4 \mathrm{~m} \mathrm{~s}^{-1}$. The encounters with P10 were evidently closer than with the other two and/or when the 

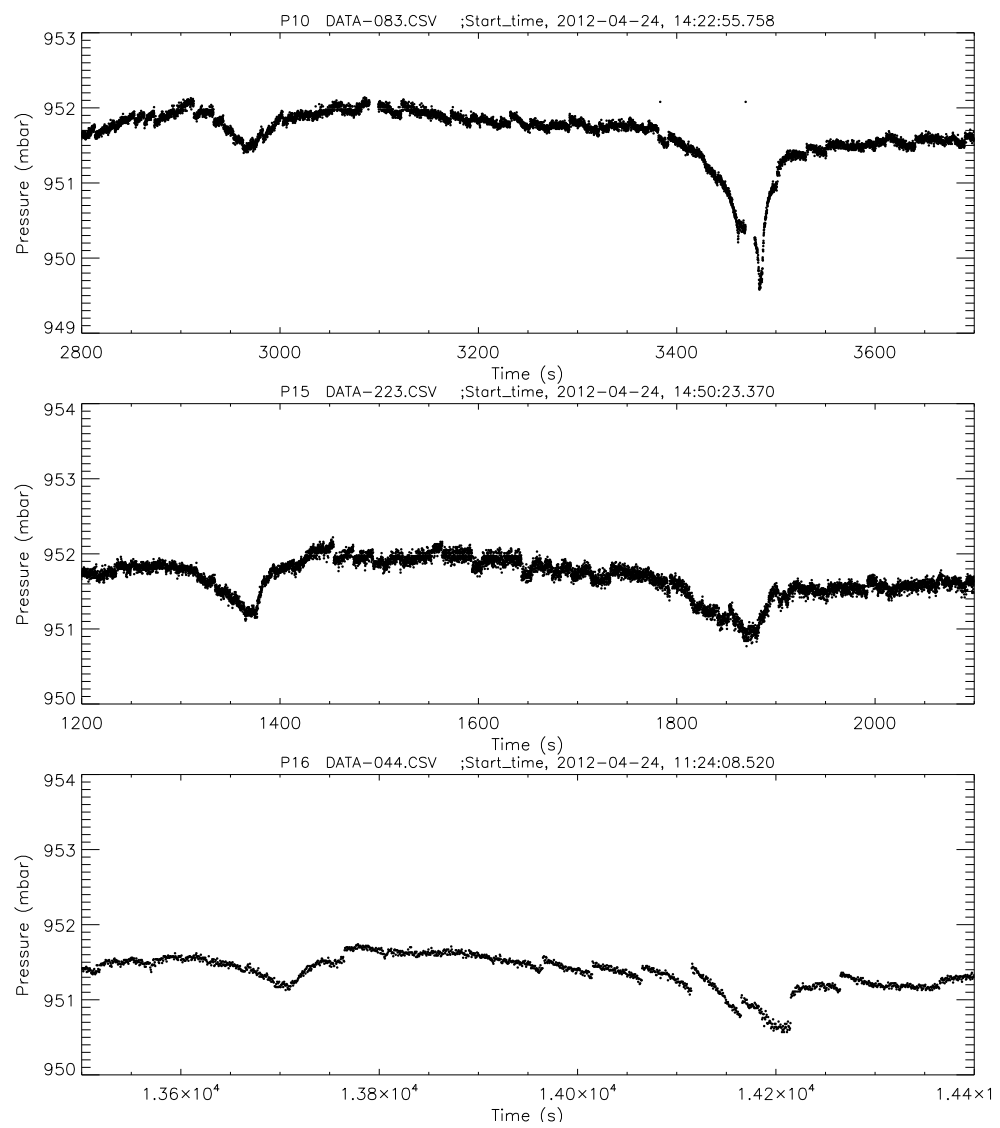

Fig. 11. A pair of dust devils, about 8 min apart, observed in all three loggers (shifted to show the coherence of the records). The bottom two plots are essentially synchronous, but the first plot is about $90 \mathrm{~s}$ earlier in time. Note that the second devil is still detectable at the $\sim 0.5 \mathrm{mbar}$ level in the last record (P16) despite the $\sim 0.25 \mathrm{mb}$ sawtooth noise (uncorrected in this plot). The second devil evidently passed closest to the P10, where the pressure drop is both sharpest in structure and deepest ( $\sim 1.5$ mbar); an $8.98 \mathrm{~s}$ gap exists in the data at $3469.496 \mathrm{~s}$, in the middle of the dust devil encounter, suggesting a possible electrostatic interference.

devils were more intense. Since the P15 dip appears deeper than P16, at least for the first devil, it seems likely that the devil passed closer to P15 than to P16.

Both dust devils, in all three records, appear to be asymmetric, with a slower dip and fast recovery. The possibility (discussed above) that this could be caused by "death" of the dust devil after P10 passage appears to be excluded in this instance in that an apparently correlated encounter with P15/16 occurs. A random encounter of a new devil appears unlikely. It is clear that multiple stations offer interesting prospects for array study, and even with only 3 stations important additional information is obtained and can address ambiguities inherent in single-station records.

Note that there is some evidence of a dual dip in the second devil, perhaps indicating the core. Interestingly, the P16 record has a data gap here where no data was written (while data gaps do occur briefly when a new datafile is being opened, in this instance the gap appears in the middle of a file). It is possible that some electrostatic discharge effect was responsible.
A final observation is noted here, which, while not a direct dust devil observation, may be related. A pseudoperiodic pressure cycle often emerges in the afternoon, with a characteristic period of the order of $1000 \mathrm{~s}$ (see Fig. 12). A similar periodicity was noted by Renno et al. (2004) in soil heat flux data in a dust devil field campaign in Eloy, Arizona, in May 2002. Variations of about $30 \%$ in the soil heat flux were noted with a period of about 20 minutes (see their Fig. 5 - although the text and caption says $30 \mathrm{~min}$, the peak spacing is more accurately expressed as $20 \mathrm{~min}$ ). Renno et al. (2004) speculate that a radiative feedback may be involved, wherein strong surface heating prompts vigorous convection and dust-raising: dust scatters and absorbs sunlight, reducing surface heating and stabilizing the atmosphere to suppress convection; once the dust settles out or is advected away, surface heating begins again. The coincidence of the $20 \mathrm{~min}$ period here with that of Renno et al. (2004) is intriguing. The phenomenon will be investigated further with a more robust dataset. 

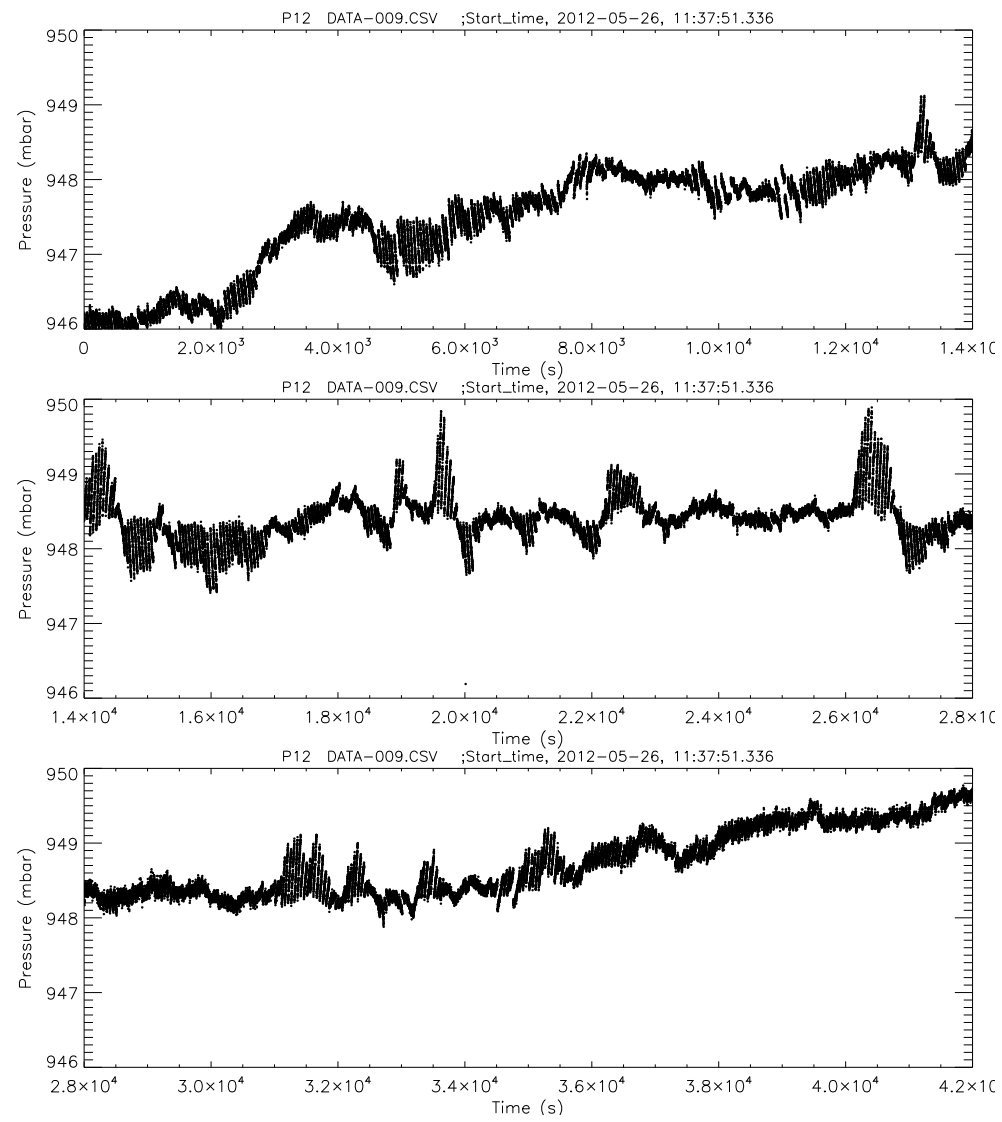

Fig. 12. Uncorrected pressure record over daylight hours. Beginning around 13000 s (i.e., around local noon), an oscillation in pressure develops with a period of about $1000 \mathrm{~s}$, and is seen most prominently at the middle of the center panel at around 14:00 LT when dust devil activity is near its peak. In this uncorrected data, the periodic pressure cycle is somewhat highlighted by the sawtooth due to correlated temperature changes.

\section{Conclusions and future work}

The unit investigated here is affordable enough $(\$ 120)$ to consider deploying in arrays to measure the two-dimensional structure of convection, or (with wider spacing) simply to increase the number of dust devil encounters obtained. The unit cost is modest enough compared with the costs of deployment that it can be considered partially expendable (i.e., some attrition of a deployed set can be tolerated with useful data still obtained).

The data storage capability of the units permits several months of data at a high sample rate $(10 \mathrm{~Hz})$ or a year or more at a lower rate. The default AA battery installation, however, permits less than a week at this rate. The power supply modifications described in this paper enable much longer-term operation than the nominal configuration, and thus permit a larger amount of data to be acquired, permitting (for example) robust statistics over several months to be obtained from a single deployment in the field.

Our initial surveys highlighted that solar-powered installations may suffer higher attrition than more covert battery-powered units. Additionally, the importance of temperature correction of the pressure data is noted: specifically, the temperature of the sensor should be logged (and thus the correction updated) at a cadence different from the duration of events being searched for. Although a data volume penalty results, this cadence should be as high as possible, once a second or better. We have described, however, how data degraded by less frequent $(\sim 50 \mathrm{~s})$ updates can be substantially recovered by post-processing.

We have identified a number of dust devil signatures in the data, finding that dust devils with pressure drops of $\sim 0.3$ mbar or larger are easily identified, and so far one 1.5 mbar event has been seen. Although this is not as deep as the largest drops measured by vehicle-borne measurements penetrating the core of devils, the record is unbiased by vehicle movement and unaffected by engine vibration. One double-devil event is detected by three different stations, showing the potential for array measurements. An interesting periodic pressure fluctuation has been noted in the convectively-active afternoon, comparable with a similar 
temperature/flux variation noted by other workers at dust devil sites.

In future work we will examine the data from ongoing field measurements, using both manual and automated methods, to derive an in-situ terrestrial dust devil census comparable with those done at Mars. Such analysis will need to take into account a time-variable noise background; with the improved temperature sampling it seems likely that a detection limit of better than 0.2 mbar may be achieved. The large number of encounters expected will permit statistically robust studies of periodicity/clustering, and an evaluation of the size statistics (Lorenz, 2012). Data from sensors such as these can be augmented by comparison with other meteorological variables such as wind speed.

Acknowledgements. This work was funded by NASA through the Mars Fundamental Research Program. Brian Jackson, Pete Lanagan and Jani Radebaugh are thanked for assistance in the field. I thank Alex Kooney of GCDC for useful correspondence regarding internal details of the dataloggers. I thank the several reviewers for their comments.

Edited by: A. Benedetto

\section{References}

Balme, M. and Greeley, R.: Dust devils on Earth and Mars, Rev. Geophys., 44, RG3003, doi:10.1029/2005RG000188, 2006.

Bosch Sensortech: BMP-085 Digital Pressure Sensor, Product Datasheet rev 1.2, Robert Bosch GmbH, Germany, 2009.

Ellehoj, M. D., Gunnlaugsson, H. P., Taylor, P. A., Kahanpaa, H., Bean, K. M., Cantor, B. A., Gheynani, B. T., Drube, L., Fisher,
D., Harri, A.-M., Holstein-Rathlou, C., Lemmon, M. T., Madsen, M. B., Malin, M. C., Polkko, J., Smith, P. H., Tamppari, L. K., Weng, W., and Whiteway, J.: Convective vortices and dust devils at the Phoenix Mars mission landing site, J. Geophys. Res., 115, E00E16, doi:10.1029/2009JE003413, 2010.

GCDC: Barometric Pressure USB Data Logger Model B1100-1, Product Manual, Gulf Coast Data Concepts, Alabama, 2010.

Lambeth, R. L.: On the measurement of dust devil parameters, B. Am. Meteorol. Soc., 47, 522-526, 1966.

Lorenz, R. D.: Pressure Drops in Dust Devils: Earth and Mars, Planet. Space Sci., 60, 370-375, 2012.

Lorenz, R. D., Jackson, B., and Barnes, J.: Inexpensive Timelapse Digital Cameras for Studying Transient Meteorological Phenomena: Dust Devils and Playa Flooding, J. Atmos. Ocean. Tech., 27, 246-256, 2010.

Murphy, J. and Nelli, S.: Mars Pathfinder convective vortices: frequency of occurrence, Geophys. Res. Lett., 29, 2103, doi:10.1029/2002GL015214, 2002.

Pathare, A. V., Balme, M. R., Metzger, S. M., Spiga, A., Towner, M. C., Rennò, N. O., and Saca, F.: Assessing the power law hypothesis for the size-frequency distribution of terrestrial and Martian dust devils, Icarus, 209, 851-852, 2010.

Renno, N. O., Abreu, V. J., Koch, J., Smith, P. H., Hartogensis, O. K., De Bruin, H. A. R., Burose, D., Delory, G. T., Farrell, W. M., Watts, C. J., Garatuza, J., Parker, M., and Carswell, A.: MATADOR 2002: A pilot field experiment on convective plumes and dust devils, J. Geophys. Res., 109, E07001, doi:10.1029/2003JE002219, 2004.

Ryan, J. A. and Lucich, R. D.: Possible Dust Devils, Vortices on Mars, J. Geophys. Res., 88, 11005-11011, 1983.

Sinclair, P. C.: The Lower Structure of Dust Devils, J. Atmos. Sci., 30, 1599-1619, 1973.

Wyett, R. E.: Pressure Drop in a Dust Devil, Mon. Weather Rev., $82,7-8,1954$. 